\title{
CIRCUITOS EN UN ESPACIO NOMBRADO: TOPONIMIA Y CONOCIMIENTOS ETNOECOLÓGICOS QOM
}

\author{
Circuits in a named space: \\ toponimy and ethnoecological kwnowledge among Toba people
}

Florencia Tola* y Celeste Medrano*

\section{Resumen}

Los qom (o tobas) conforman un grupo indígena chaqueño descripto como cazador-recolector. Numerosos textos documentaron las actividades de subsistencia propias de este grupo. Fuentes de comienzo del siglo XX, refieren a la existencia de recorridos dentro de los territorios indígenas que, visitados sucesivamente, delineaban las actividades de caza, pesca y recolección. Autores contemporáneos describieron diversos aspectos de la toponimia y del territorio. En nuestro trabajo de campo etnográfico en comunidades del centro-sur de Formosa hemos registrado no sólo una abundante toponimia, sino también información novedosa sobre circuitos, antiguos y actuales, que unían los sitios nombrados. Dichos circuitos también poseen nombres así como un trazado particular. Este último aspecto alude a una regulación en el aprovechamiento de las especies empleadas para la caza y la pesca. Este artículo se propone debatir con las diversas posiciones que describen el modo que tenían los qom de relacionarse con los recursos naturales y analiza de manera interrelacionada la movilidad espacial asociada a la subsistencia y a los conocimientos etnoecológicos tobas.

$$
<\text { movilidad }><\text { toponimia }><\text { etnoecología }><\text { tobas }>
$$

\begin{abstract}
Qom (or Toba) people are one of the hunter-gatherers groups of the Gran Chaco. Different texts documented subsistence activities of this group. Sources of beginning of XXth century, refer to the existence of circuits within the indigenous territories that were visited successively and delineated the hunting and gathering activities. Contemporary authors described diverse aspects of the toponymy and the territory of Toba people. In our fieldwork in communities of the center-south of Formosa province (Argentinean Chaco), we registered not only an important toponymy, but also information about the ancient and present circuits that united the named places. These circuits have also a name and a specific drawing. This last aspect refers to a regulation of the species hunted and fished. This paper describes, in an interrelated way, ethno-ecological knowledge and mobility among Toba people.
\end{abstract}

$<$ mobility $><$ toponimy $><$ ethnoecology $><$ Toba people $>$

Recibido: 03/04/2014 // Aceptado: 15/07/2014

\footnotetext{
* Doctora en Antropología Social y Etnografía, Investigadora Adjunta CONICET, tolatoba@yahoo.com

* Doctora en Antropología, Becaria Post Doctoral CONICET, Inst. de Ciencias Antropológicas UBA,celestetazo@hotmail.com
} 
Tola y Medrano. Circuitos en un espacio nombrado: Toponimia y conocimientos...

\section{Presentación}

Los circuitos antiguos y actuales de marisca (caza, pesca, meleo y recolección) de los tobas $(q o m)^{1}$ constituyen un tema sobre el cual hay bastante por ahondar, más aún si se los vincula con el aprovechamiento de los recursos naturales, con la historia oral de las comunidades y con una cosmología en la que el universo está poblado por una variedad de seres no-humanos (entre ellos, los dueños de animales, vegetales y ámbitos) con los cuales los humanos se relacionan². En este artículo dejaremos de lado la cuestión cosmológica para adentrarnos en aspectos de la movilidad espacial (circuitos de marisca) vinculados con la micro-historia regional y con los conocimientos etnoecológicos de los tobas de algunas comunidades del centro-sur de la provincia de Formosa (Km 503, San Carlos, Riacho de Oro y Tres Pozos, ver Figura 1).

Los actuales habitantes de estas comunidades descienden de miembros de cuatro antiguas tribus guaycurúes (huaguilot, no'olgaxanaq, rapicoshec y ỹolo) que antes de la conquista del Chaco recorrían un amplio territorio a ambas márgenes del río Bermejo ${ }^{3}$. En las genealogías realizadas en las cuatro comunidades registramos por lo menos ocho generaciones, y en los relatos de los ancianos nacidos a comienzos del siglo $\mathrm{XX}$ se escucha que sus ascendentes en tres generaciones recorrían la zona actual de las comunidades, considerada como $s u$ lugar dentro de un territorio más amplio.

El territorio de estas antiguas tribus comprendía lugares nombrados a lo largo de las actuales provincias de Chaco y Formosa que, si bien podrían ser considerados como dispersos, se encontraban y se encuentran articulados a partir de amplios y antiguos circuitos estacionales, y de reducidos y actuales recorridos de marisca. Los circuitos estacionales remiten a grandes circuitos por un amplio territorio que los miembros de las antiguas bandas y tribus recorrían en función de las estaciones del año y la disponibilidad de recursos, mientras que los recorridos de marisca son más reducidos y son transitados por los actuales qom durante sus diversas actividades de subsistencia.

\footnotetext{
'El Gran Chaco constituye una vasta llanura con porciones boscosas que abarca parte de Argentina, Bolivia, Paraguay y Brasil. Limitada al este por los ríos Paraná y Paraguay, al oeste por la pre-cordillera de los Andes, al norte por los Llanos de Chiquitos y la meseta del Mato Grosso y al sur por el río Salado, comprende un área de más de $1.000 .000 \mathrm{~km}^{2}$ (Morello et al., 2009). Los tobas, pertenecen -junto con otros grupos chaqueños como los pilagás y los mocovíes- a la familia lingüística Guaycurú y se autodenominan qom.

2 Sobre los seres no-humanos, los dueños de animales, plantas y ámbitos, la cosmología qom en general, $c f$. Wright (2008), Tola (2009, 2010, 2012), entre otros.

3 Varios autores (Karsten, 1932; Métraux, 1963[1944]; Kersten, 1968; Cordeu y De los Ríos, 1982:163; Braunstein, 1983) coinciden en que la unidad básica de la organización social en el Chaco fue la 'banda'. Éstas estaban constituidas a partir de la unión de familias extensas cuyos miembros se inscribían en un entramado de alianzas que los incluía a casi todos (Cordeu y de los Ríos, 1982; Braunstein y Miller, 1999). Las bandas se desplazaban por territorios más o menos definidos al ritmo de los ciclos ecológicos, constituyéndose a partir de una localización compartida. Las tribus eran, en cambio, unidades políticas menos localizadas que agrupaban a varias bandas aliadas en torno a una misma variante dialectal y a relaciones de parentesco a través del intercambio (Karsten, 1932; Cordeu y De los Ríos, 1982; Braunstein, 1983).
} 
Figura 1. Comunidades qom donde realizamos trabajo de campo

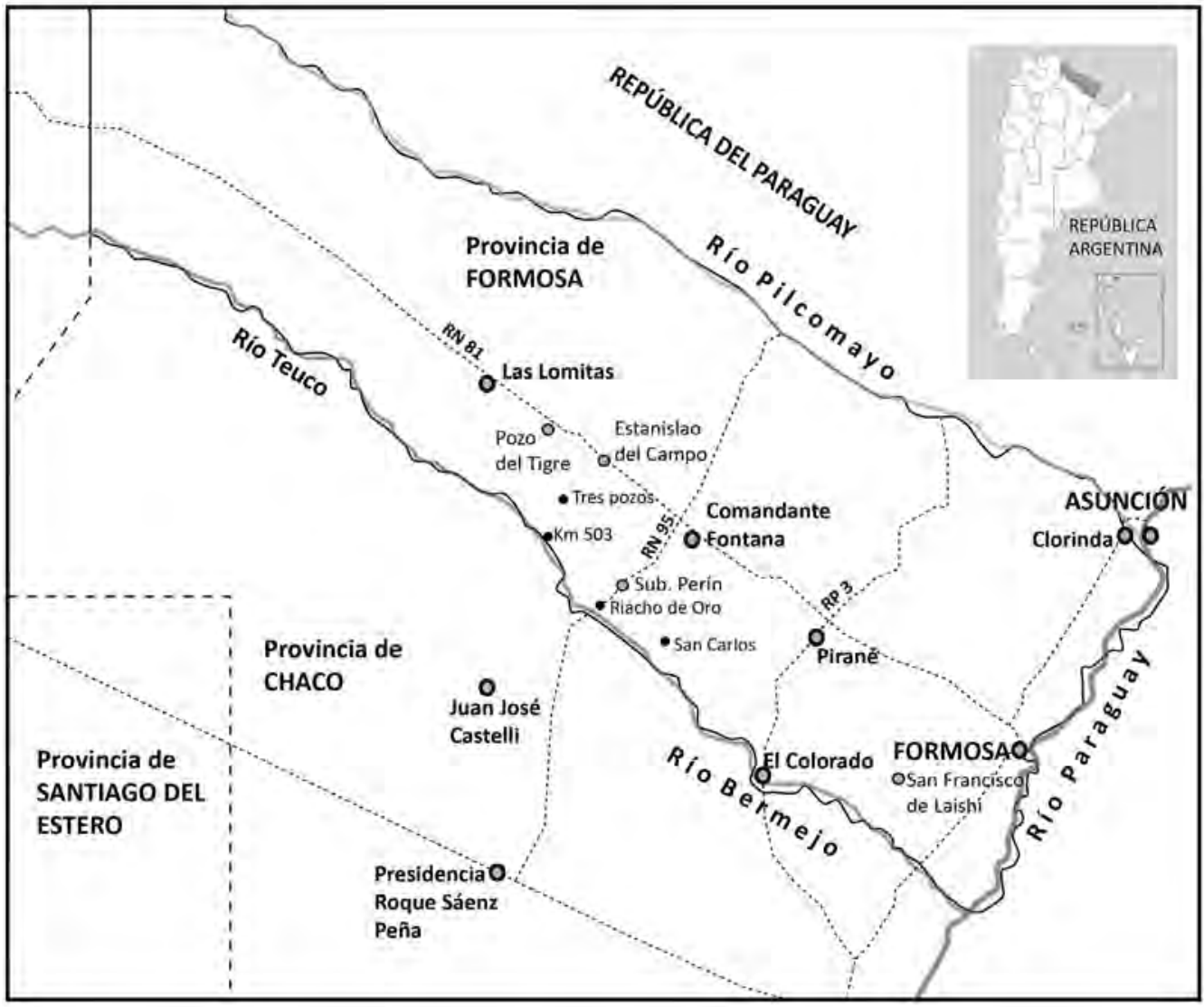

En este trabajo nos referiremos a los conocimientos etnoecológicos de los tobas de la zona mencionada, entendidos como aquellos conocimientos específicos relativos al aprovechamiento de la flora y la fauna, y también como los saberes y la praxis del entorno en términos generales. Mostraremos que estos conocimientos etnoecológicos se hallan histórica y geográficamente situados en la medida en que están asociados a las unidades sociales que, a lo largo de las sucesivas generaciones, los fueron generando en su relación con el entorno.

Víctor Toledo (1992: 6), uno de los principales referentes de la etnoecología etnociencia más reciente respecto de aquellas nacidas a finales del siglo XIX ${ }^{4}$-, sostiene que la etnoecología es el estudio interdisciplinario de la percepción que diversos grupos

\footnotetext{
4 Desde la década del 60, se vienen produciendo trabajos etnocientíficos sobre los tobas; ya sea de corte etnobotánico (Martínez Crovetto, 1964; Vuoto, 1981, 1999; Arenas, 2003; Martínez, 2007, 2008, 2009 a y b, 2010), ya sea de corte etnozoológico (Vuoto, 1981 a y b; Martínez Crovetto, 1995; Cúneo y Porta, 2009; Arenas y Porini, 2009; Medrano et al., 2011; Medrano, 2012, 2013, 2014). No abundan, sin embargo, abordajes específicamente vinculados a la etnoecología, aunque sí existen producciones que adoptan una visión holística del tema (cf. Martínez, 2011, 2013).
} 
Tola y Medrano. Circuitos en un espacio nombrado: Toponimia y conocimientos...

humanos tienen de la naturaleza. El autor enfatiza que dicha percepción incide en el uso, el manejo y la apropiación que los grupos hacen de los recursos naturales. Si "el kosmos (conjunto de creencias), el corpus (conjunto de conocimientos) y la praxis (conjunto de prácticas productivas)" (Toledo et al., 2001: 10) constituyen las tres dimensiones de la investigación etnoecológica, en este trabajo nos concentraremos tan sólo en el corpus y la praxis qom aun sabiendo que los mismos están permeados por un 'conjunto de creencias' o, dicho de otro modo, que ambos son parte de una cosmología particular.

Luego de realizar una sintética presentación del tema de la movilidad en la literatura chaqueña, abordaremos nuestros datos de campo acerca de la toponimia y los circuitos de marisca. Para ello, nos referiremos principalmente a la información obtenida en $\mathrm{Km} 503$ por ser allí donde registramos, además de la toponimia y los recorridos actuales, los antiguos circuitos nombrados. En San Carlos, Riacho de Oro y Tres Pozos, en cambio, obtuvimos información sobre los topónimos y los recorridos de marisca pero no encontramos, hasta el momento, evidencia de la existencia de circuitos nombrados.

\section{Movilidad y organización social chaqueña}

La historia de los grupos indígenas chaqueños se caracteriza por diversos tipos de movilidades, entre las que se destacan los recorridos de marisca, el abandono de zonas de ocupación por factores sociales, las migraciones fuera del Chaco, las relocalizaciones y las migraciones estacionales y laborales. La conquista y colonización del Chaco argentino generaron cambios significativos en los patrones de movilidad de los grupos indígenas y dieron lugar a una nueva geopolítica en la que los indígenas quedaron relegados a zonas reducidas, alejadas y desventajosas en términos de recursos.

Desde los comienzos del siglo XX, la literatura chaqueña brinda referencias a la movilidad de los grupos indígenas, asociada tanto a los recursos naturales como a factores sociológicos y a otros ligados al contacto con la sociedad mayor. Guido Boggiani (1900), por ejemplo, vinculaba la movilidad con las actividades de subsistencia; Rafael Karsten (1932) detallaba las "condiciones naturales" (inundaciones, escases de pasturas, de agua y presas) y se refería también a motivos sociales y religiosos (el deseo de recorrer y de encontrarse con parientes o amigos así como razones supersticiosas). A su vez, Enrique Palavecino (1936: 444) se refería a la movilidad diaria de los indígenas señalando que ésta tenía un orden y que cada punto cardinal era objeto de exploraciones sistemáticas. Alfred Métraux (1996[1946]: 26), por su parte, además de remitir a factores de orden ecológico (abundancia de especies vegetales y animales) mencionaba los viajes de los chaqueños a las plantaciones de azúcar.

Las etnografías contemporáneas coinciden en que antes de la conquista y colonización, las bandas y tribus guaycurúes se fisionaban durante el período invernal y se fusionaban en la estación cálida, conformando éstos los dos grandes momentos del ciclo anual. Al respecto, Marcela Mendoza $(2002,2003-2004)$ señala que las bandas tobas del oeste formoseño se fusionaban o fisionaban en función de la disponibilidad estacional de recursos vinculados a la flora, la fauna y el agua: "During the most aggregated phases, several bands (each of more than 100 individuals) would get together in large camps to 
take advantage of winter fishing or to gather algarrobo beans at the beginning of summer" (2003-2004: 6). José Braunstein (1993, 2012) diferencia los grupos chaqueños con bandas semi-móviles que tenían una movilidad ritmada por el ciclo anual y cuyos integrantes no se separaban de manera regular durante éste, de aquellos grupos con bandas semipermanentes que poseían una morfología doble regida por el ritmo estacional. En estos últimos grupos de carácter más inestable, los integrantes de las familias extensas se separaban durante cierto período del año y luego se volvían a agrupar por lo general en un lugar fijo (Braunstein, 2012). Esta migración estacional habría estado marcada por el ritmo de las condiciones ecológicas:

El concreto ciclo estacional de una de estas familias o bandas, describía desde tiempo inmemorial un circuito sobre el territorio enmarcado entre dos polos en los que permanecían más tiempo: en invierno, cerca del río y en verano, en el interior. El camino aparece jalonado por puntos de parada intermedios más breves junto a las aguadas. Cada punto de detención coincidía aproximadamente con el género de actividad propia del momento del ciclo en que se hallaba la región (2012: 7).

Pablo Wright (2003b) si bien recopiló relatos orales referidos a la existencia de rutas indígenas y circuitos de marisca, no profundizó en el análisis de su cuerpo documental. Al respecto, escribió: "los mariscadores descansaban en nmachaqa' [campamentos] ya conocidos de antemano, por mentas o por experiencia propia. Estos se ubicaban casi siempre en lugares intermedios en las rutas de marisca, lo que permitía organizar mejor la marcha y evitar el cansancio excesivo" (2003b: 113, resaltado nuestro).

A nivel regional son escasos los modelos interpretativos que muestran el modo en que las antiguas unidades sociales o las actuales comunidades organizaban y organizan sus recorridos de marisca o sus circuitos más amplios a través de los lugares nombrados. Este último tema, la toponimia, suscitó el interés de varios especialistas contemporáneos quienes registraron lugares nombrados y explicitaron también los criterios de la clasificación y nombramiento de los mismos ${ }^{5}$.

A continuación, a partir de nuestros datos de campo, trataremos de articular los circuitos y los topónimos con aspectos de la organización social y los conocimientos etnoecológicos tobas.

\footnotetext{
5 Miller (1979), Dell'Arciprete (1991), Wright (1991, 2003b), Braunstein (1993), Palmer (1995), De la Cruz (1995a y b), Censabella (2009), Fernández y Braunstein (2001), Salamanca (2011), Tola, Medrano y Cardin (2011), entre otros.
} 
Tola y Medrano. Circuitos en un espacio nombrado: Toponimia y conocimientos...

\section{Hilvanando topónimos}

$\underline{K m 503}$

La comunidad Km 503, llamada en toba Lapel saq emapec Loxolqai' se encuentra ubicada sobre el río Bermejo, a $45 \mathrm{~km}$ del pueblo Estanislao del Campo. Posee una superficie de 260 hectáreas, aunque los tobas que allí residen aún carecen del título de propiedad comunitaria que es habitualmente otorgado por el Instituto de Comunidades Aborígenes de la provincia de Formosa. Hoy en día Km 503 cuenta con una población de 50 habitantes que descienden de individuos que pertenecían a las tribus huaguilot, yolo y rapicoshec. La cantidad de habitantes de Km 503 ha ido fluctuando debido a factores de orden social, político y ambiental. Todas las familias de $\mathrm{Km} 503$ cruzan permanentemente el río y pasan parte del año en las comunidades de la zona de El Espinillo en donde residen sus parientes. Asimismo, muchas de ellas cobran sus pensiones, planes sociales y jubilaciones en la provincia de Chaco, razón por la cual mes a mes van a Chaco para realizar sus cobros y trámites varios. Por otro lado, los habitantes de Km 503 se encuentran desde hace años en tensión con sus vecinos criollos que no respetan la mensura comunitaria e ingresan en sus tierras avasallando sus casas y zonas de marisca ${ }^{6}$. Asimismo, en la zona se verifican crecidas del río Bermejo que impulsan a los qom a dejar momentáneamente la comunidad.

En 2011 comenzamos un proyecto sobre el territorio y la memoria en esta comunidad y en Riacho de Oro y Tres Pozos, y durante cinco viajes de campo (de aproximadamente 15 días por viaje) registramos información genealógica, saberes etnoecológicos, la historia oral de los desplazamientos de los antepasados e historias de marisca ${ }^{7}$. Si bien hemos trabajado en las tres comunidades mencionadas, nos decidimos concentrar en Km 503 por ser ésta una comunidad en la que sus habitantes subsisten gracias a la marisca y por no existir, hasta el momento, mayor información etnográfica y etnoecológica sobre la misma ${ }^{8}$. En un primer momento, a la par que registrábamos mediante entrevistas bilingües y conversaciones informales tanto en la comunidad como recorriendo el territorio, la historia del uso del territorio, de las reiteradas ocupaciones de Km 503 y las prácticas de marisca, confeccionamos un primer mapa (Figura 2) en el que plasmamos la ubicación actual de Km 503 y los antiguos sitios de residencia.

\footnotetext{
${ }^{6} \mathrm{Km} 503$ atraviesa diversos tipos de conflictos territoriales. Por un lado, la comunidad no cuenta con el título definitivo de propiedad comunitaria a pesar de que, desde hace varios años, posee personería jurídica y se le ha hecho la mensura del perímetro comunitario. Por otro lado, varios vecinos criollos se han asentado desde hace algunos años en las tierras que, si bien no figuran en la mensura realizada por los agrimensores del I.C.A, son utilizadas por los qom en sus actividades de subsistencia. Asimismo, estos vecinos suelen no respetar la mensura comunitaria e invadir la propiedad de los qom, haciendo pastar sus animales y colocando cercos y candados en las tierras indígenas.

7 Proyecto financiado por UNESCO-IESALC (junio-noviembre 2011): "Territorio, memoria y lengua entre los qom del centro-este de la provincia de Formosa en el Chaco argentino". El equipo estaba conformado por Florencia Tola, Celeste Medrano, Lorena Cardin y Gala Coconier, y contaba con el respaldo de Valentín Suarez, líder de Riacho de Oro, quien además de facilitarnos el acceso a Km 503 por conocer allí a sus habitantes, colaboró en las traducciones, dibujos y registro de toda la información.

8 La información que brindamos de San Carlos fue obtenida en trabajos de campo previos a los realizados en el marco del proyecto mencionado.
} 
Figura 2. Topónimos y comunidad Km 503

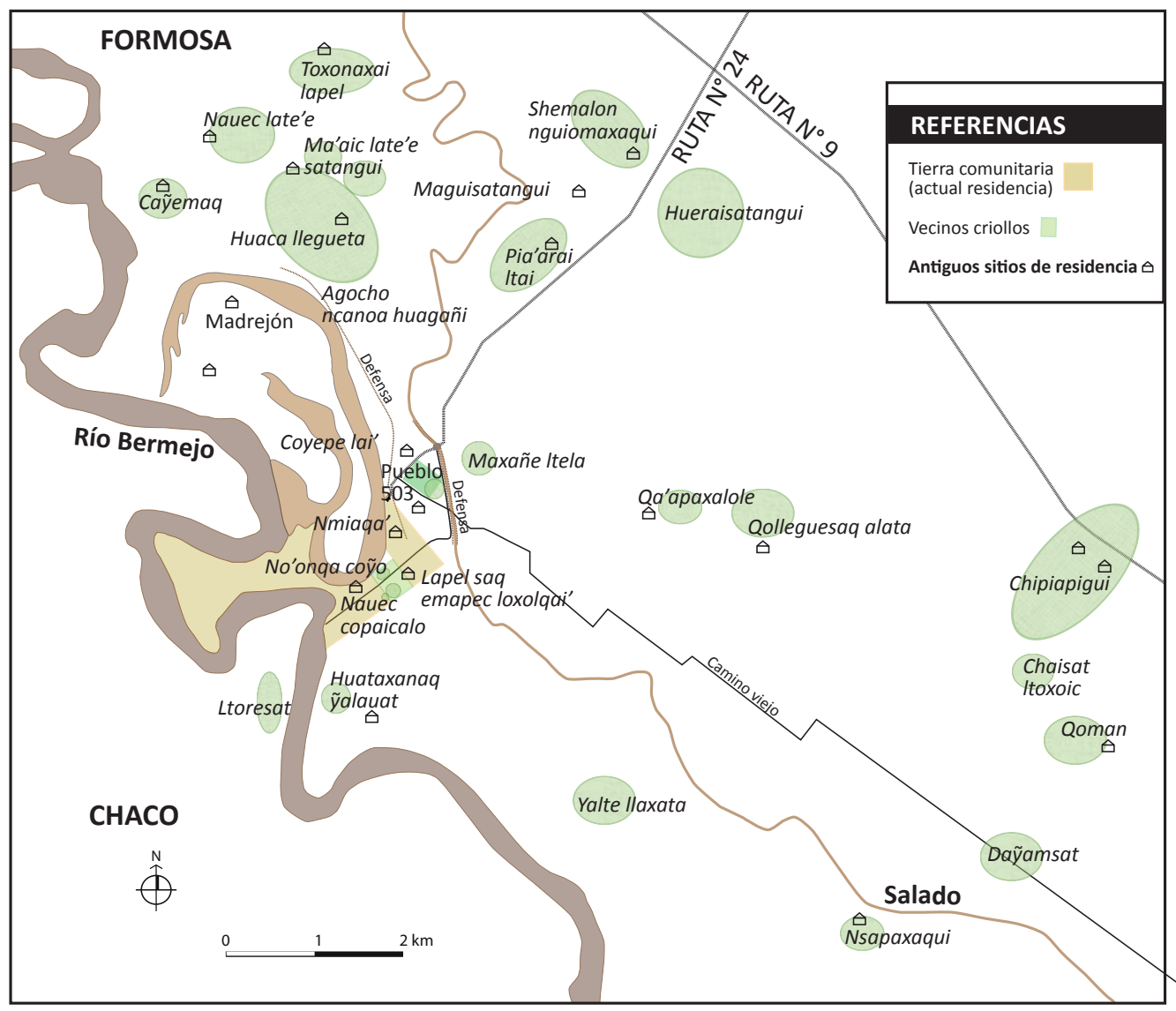

Posteriormente, en una de las estadías de campo, mientras trabajábamos sobre el antiguo territorio con los ancianos de la comunidad, el líder de la misma (cazador y chamán) mencionó la existencia de circuitos de marisca que poseen nombre. Hasta el momento, registrábamos la mayor cantidad posible de topónimos, pero no los visualizábamos en tanto lugares que, en su conjunto, conformaban circuitos $\mathrm{y}$, menos aún, circuitos nombrados. Éstos eran reconocidos por los antiguos y por los actuales qom de la zona y con el tiempo identificamos que ellos conformaban parte de su microhistoria.

Fue así que comenzamos un registro más minucioso con los líderes de $\mathrm{Km} 503$ y Riacho de Oro, con todos los cazadores de $\mathrm{Km} \mathrm{503,} \mathrm{con} \mathrm{las} \mathrm{recolectoras} \mathrm{y} \mathrm{con} \mathrm{los}$ ancianos y ancianas que recordaban o habían transitado dichos circuitos. A partir de ese momento, los lugares nombrados se ordenaron como parte de un sistema mayor de caza, pesca, recolección, meleo y encuentros de sociabilidad entre las tribus chaqueñas mencionadas. 
No sólo registramos los nombres de los circuitos y los lugares nombrados que ellos articulan, las historias asociadas a cada uno, sino también indagamos acerca de si eran lugares de campamento por varios meses o de ida en el día, registramos los recursos que se encontraban allí, la época del año en la que se lo visitaba y los acontecimientos memorables trasmitidos generación por generación. Recorrimos también casi todos los lugares que conforman dichos circuitos; algunos cercanos y otros a más de 5 horas de distancia en canoa, caminata o moto.

A continuación, describiremos estos circuitos nombrados y mencionaremos tan sólo algunos topónimos, para discutir luego aspectos de la articulación entre el territorio y la subsistencia.

\section{Circuitos nombrados de la zona de Km 503}

Hemos registrado tres circuitos nombrados en la zona. Haciendo uso de las denominaciones de los criollos, varios qom refirieron que estos circuitos equivaldrían a las "rutas nacionales" por su extensión y por el hecho de ser conocidos por muchos qom de otras comunidades chaqueñas además de los habitantes de Km 503, Riacho de Oro, San Carlos y Tres Pozos. En la Figura 3 presentamos la totalidad de los topónimos registrados: 
Figura 3. Topónimos y circuitos

\begin{tabular}{|c|c|c|c|c|}
\hline \multirow[t]{2}{*}{ Topónimo } & \multirow[t]{2}{*}{ Traducción } & \multicolumn{3}{|c|}{ Recorrido del que participa } \\
\hline & & Roquiaxanaxat & Nchaxanaxat & Pela'ate' \\
\hline $\begin{array}{l}\text { Agocho ncanoa } \\
\text { huagañi }\end{array}$ & $\begin{array}{l}\text { donde se le dio vuelta la canoa a } \\
\text { Agocho }\end{array}$ & & & \\
\hline caỹemaq & caballo ahorcado & $\mathrm{x}$ & & \\
\hline chaisat ltoxoic & palmar antiguo & & $\mathrm{x}$ & \\
\hline chipiapigui & donde chispea & & $\mathrm{x}$ & \\
\hline coyepe lai' & donde abunda el chimuco (ave s/i) & & & \\
\hline daỹamsat & $\begin{array}{l}\text { donde abunda la fruta del cactus } \\
\text { (tunal) }\end{array}$ & & $\mathrm{x}$ & \\
\hline elaxarae' la'alqa & donde se asustó & & & $\mathrm{x}$ \\
\hline huacallegueta & cruce de las vacas & $\mathrm{x}$ & & \\
\hline huataxanaq ỹalauat & el policía mató & & $\mathrm{x}$ & \\
\hline hueraisatangui & $\begin{array}{l}\text { donde abunda la 'vieja del agua' } \\
\text { (LORICARIDAE) }\end{array}$ & & $\mathrm{x}$ & \\
\hline $\begin{array}{l}\text { lapel saq emapec } \\
\text { Loxolqai' }\end{array}$ & $\begin{array}{l}\text { laguna que nunca se seca de } \\
\text { Loxolqai' }\end{array}$ & & $\mathrm{x}$ & \\
\hline ltoresat & $\begin{array}{l}\text { bobadal quemado (de palo bobo: } \\
\text { Tessaria integrifolia) }\end{array}$ & & $\mathrm{x}$ & \\
\hline ma'aic late'e satangui & donde abunda ma'aic late'e & $\mathrm{x}$ & & \\
\hline maguisatangui & donde abunda el sauce (Salix sp.) & $\mathrm{x}$ & & \\
\hline maxañe ltela & oreja doblada hacia abajo & & $\mathrm{x}$ & \\
\hline nauec copaicalo & $\begin{array}{l}\text { bosque de gato onza (Leopardus } \\
\text { pardalis) }\end{array}$ & & $\mathrm{x}$ & \\
\hline nauec late'e & bosque grande & $\mathrm{x}$ & & \\
\hline nmiaqa' & lugar de 'baile sapo' & $\mathrm{x}$ & $\mathrm{x}$ & $\mathrm{x}$ \\
\hline no'onqacoỹo & lugar lindo para los pájaros & & $\mathrm{x}$ & \\
\hline nsapaxaqui & donde se constipó & & $\mathrm{x}$ & \\
\hline peraxanaxa' lamo & $\begin{array}{l}\text { tronco del palo borracho (Ceiba } \\
\text { speciosa) }\end{array}$ & & & $\mathrm{x}$ \\
\hline pia'arai ltai' & $\begin{array}{l}\text { donde murió la mujer de pie } \\
\text { defectuoso }\end{array}$ & $\mathrm{x}$ & & \\
\hline qa'apaxalole & $\begin{array}{l}\text { alojita (de aloja: chicha de } \\
\text { algarroba) (Prosopis sp.) }\end{array}$ & & $\mathrm{x}$ & \\
\hline qatec huaỹoxon & $\begin{array}{l}\text { nido flotante de lechiguana } \\
\text { (Brachygastralecheguana) }\end{array}$ & & & $\mathrm{x}$ \\
\hline qolleguesaq alata & iguana sucia (Tupinambis sp.) & & $\mathrm{x}$ & \\
\hline qoman & donde la gente está a gusto & & $\mathrm{x}$ & \\
\hline \begin{tabular}{|l|} 
Shemalon \\
nguiomaxaqui
\end{tabular} & donde devora Shemalon & $\mathrm{x}$ & & \\
\hline toxonaxai lapel & laguna que devora & $\mathrm{x}$ & & \\
\hline Yalte llaxata & el dedo de Yalte & & $\mathrm{x}$ & \\
\hline
\end{tabular}


Uno de los circuitos que registramos se denomina Roquiaxanaxat ${ }^{9}$. Este término designa una aguja de madera a la que se le ensarta por el ojo un largo hilo que usan los pescadores cuando ingresan al agua con la red (Figura 4). El hilo se ata a la cintura y, a medida que van sacando los peces del agua, se los va hincando e introduciendo en el hilo por el palo. No es casual que la raíz verbal de la que deriva el nombre instrumental del circuito sea -doqui/roqui que, según Buckwalter (2001: 39), es 'enhebrar', 'ensartar', 'enhilar' 10 .

Figura 4. Toba utilizando roquiaxanaxat

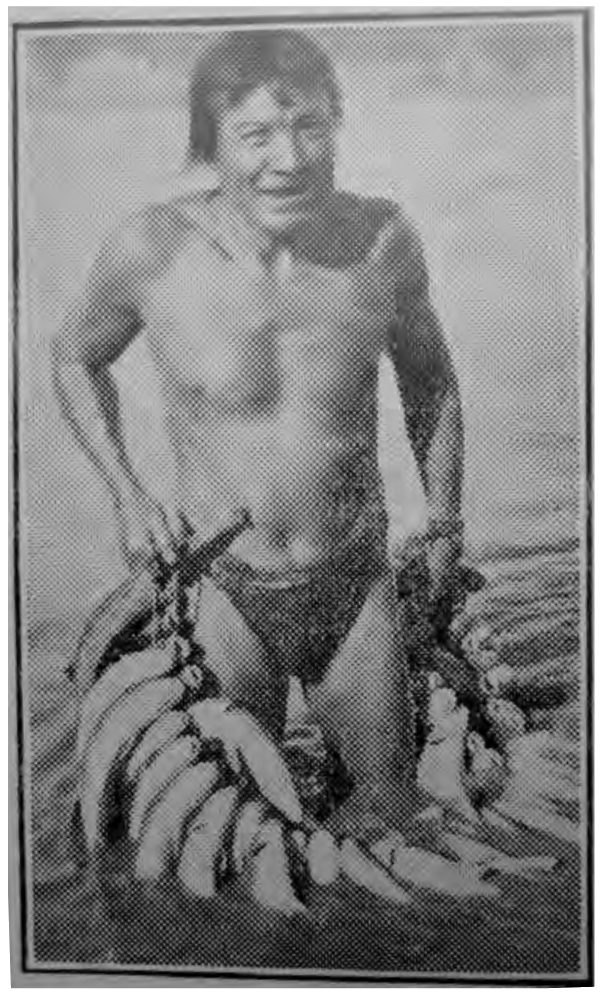

Fuente: Dickens (1938)

\footnotetext{
9 Para la transcripción de la lengua toba, seguimos la propuesta alfabética de Bulckwalter (1980).
}

${ }^{10}$ Agradecemos a Paola Cúneo por su ayuda con la identificación de la raíz verbal de este término. 
Figura 5. Circuitos antiguamente utilizados por los qom para organizar las actividades de marisca

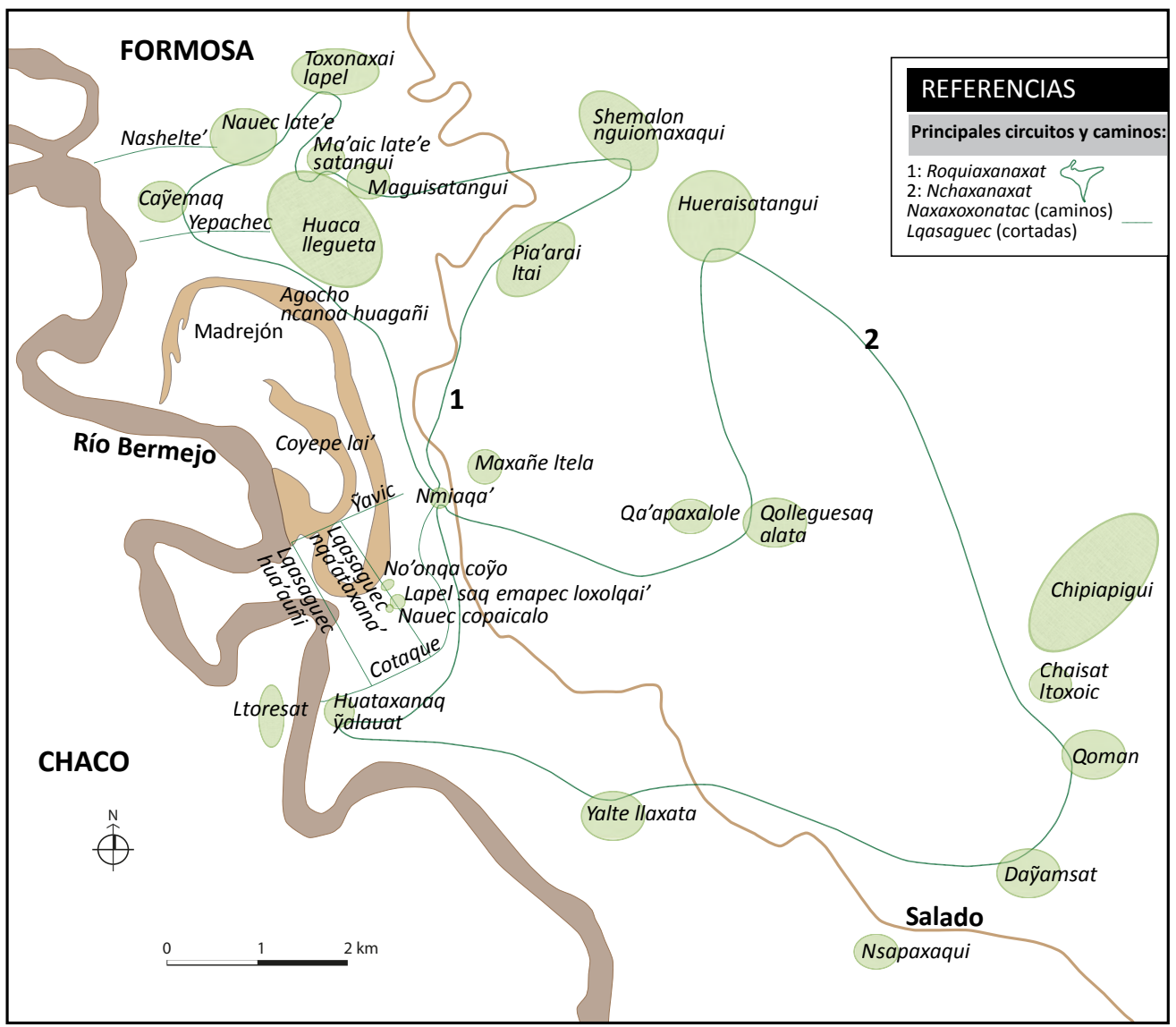

Este circuito (Figura 5) comprende lugares de pesca que se ubican desde $\mathrm{Km}$ 503 hacia el oeste: los grupos de marisca salían de nmiaqa' ${ }^{\prime 11}$ hacia caỹemaq, nauec late'e y toxonaxai lapel, y en el mes de agosto iban a huaca llegueta donde encontraban abundante pescado y aves. Allí hacían campamento y se encontraban con miembros de otras tribus con las que efectuaban intercambios, tal como se sabe por las fuentes etnográficas. De huaca entraban a dos montes próximos denominamos ma'aic late'e satangui y maguisatangui ${ }^{12}$, luego pasaban por shemalon nguiomaxaqui y pia 'arai ltai' para finalmente regresar a nmiaqa'.

${ }^{11}$ A medida que se presenten los topónimos, brindaremos la traducción de cada uno de ellos.

${ }^{12}$ Ambos topónimos llevan su nombre en función de la especie vegetal que allí domina (en estos casos Malvella leprosa -identificada en base a Martínez (2009b)- y Salix humboldtiana, respectivamente. Mediante mecanismos similares, los toba-pilagás relacionan los nombres de las plantas con unidades de vegetación de acuerdo a la fisonomía, sucesión, posibilidad de acceso y visibilidad de las mismas ( $c f$. Scarpa y Arenas, 2004). 
Tola y Medrano. Circuitos en un espacio nombrado: Toponimia y conocimientos...

Nmiaqa' es el epicentro en el cual comienzan y terminan los circuitos que describiremos. Este lugar era un centro de sociabilidad de suma importancia para estas tribus y para otras aliadas. Su nombre significa 'el lugar de baile sapo' ('baile sapo': $n m i$ ') y es conocido por gran parte de los ancianos de la zona central de Formosa y Chaco $^{13}$. Si bien todos los sitios recordados y visitados de éste y del otro circuito se corresponden con algún cuerpo de agua dulce, roquiaxanaxat reúne aquellos espacios que, por estar más cerca del Bermejo, contienen un flujo de agua más estable anualmente. Por esta razón, durante el invierno, cuando merman las lluvias, cuando se secan los ambientes acuáticos más someros y las poblaciones de mamíferos y aves aprovechables se encuentran deprimidas, los qom recorrían los lugares de roquiaxanaxat donde se dedicaban a la pesca (recurso que si bien declina parcialmente en invierno, sigue existiendo durante esta época y aporta importantes presas).

El otro circuito registrado es Nchaxanaxat. Este término remite a la soga que, apoyada sobre la frente, usan las mujeres para acarrear leña. La raíz verbal de la que deriva este nombre instrumental es -chaxan que, según Buckwalter (2001: 78), es 'llevar leña atada y suspendida de la frente' ${ }^{14}$.

Nchaxanaxat parte también de nmiaqa' pero en dirección al este y comprende huataxanat ỹalauat, yalte llaxata, qoman, daỹamsat, hueraisatangui, qolleguesaq alata para concluir y comenzar en nmiaqa'. Hasta el mes de agosto, los qom recorrían esta zona y cuando llegaba "la época de crisis" (agosto) se iban a roquiaxanaxat. Esta última información refuerza la idea de que durante la temporada cálida, los qom de esta zona recorrían los sitios de nchaxanaxat que se ubican a mayor distancia del río Bermejo y se vinculaban con ambientes tales como el monte y los palmares. En estos lugares se cazaban ñandúes (Rhea americana), mamíferos (guazunchos: Mazama sp. y pecaríes: Tayassuidae) y aves (charata: Ortalis canicollis). Las lluvias estivales renovaban el recurso dulceacuícola que se presentaba como condición sine qua non para establecer campamentos de marisca en los lugares de este circuito.

Como puede apreciarse, los dos circuitos asocian la movilidad propia de la marisca con los conocimientos que los qom poseen de las variaciones de las poblaciones de fauna a lo largo del ciclo anual. De hecho, se sabe que los qom no deambulaban por el territorio sorteando imprevistos, sino que conocían finamente la ubicación de las poblaciones animales, su abundancia y su situación reproductiva y se dirigían hacia ambientes específicos en busca de una diversidad determinada de presas. Además de los conocimientos de la fauna, ellos recorrían los circuitos en función del conocimiento que poseían de la estacionalidad chaqueña ${ }^{15}$. Ya diversos autores (De la Cruz, 1995b:

${ }^{13}$ Baile realizado por los grupos indígenas del área del Pilcomayo y del Bermejo, y cuya finalidad era, según algunas referencias, la formación de nuevas parejas.

${ }^{14}$ Agradecemos a Paola Cúneo por su ayuda con la identificación de la raíz verbal de este término.

${ }^{15}$ Actualmente, el clima chaqueño es tropical semiárido a húmedo, caracterizado por un intercambio permanente de masas de aire tropical y austral. Las temperaturas oscilan entre $\operatorname{los} 24^{\circ} \mathrm{C} \mathrm{y} 30^{\circ} \mathrm{C}$ en verano con máximas que superan $\operatorname{los} 40^{\circ} \mathrm{C}$ (Iriondo, 2006). En la zona donde hemos trabajado, el período de lluvias se corresponde con la estación cálida y se concentra durante los meses de octubre a abril. El mínimo de precipitaciones se registra durante la estación invernal, entre los meses de junio a agosto, en 
77; Wright, 2003b: 551; Mendoza, 2003-2004; Braunstein, 2012) expresaron que la circulación y los asentamientos dependían de las fuentes de agua asociadas al régimen pluvial. Al respecto, Braunstein refiere que "las actividades de producción tradicionales estaban ritmadas por las condiciones estacionales y por la existencia consecuente, en determinados momentos, de ciertos bienes en las diversas partes del territorio" (ibid.: 6). Guillermo Lamenza señala, por su parte, que "la situación hidrológica regional hace que el agua sea un recurso crítico, de difícil acceso, que requiere de un manejo especial por parte de las poblaciones humanas" (2013: 16). En nuestro caso, todos los topónimos que registramos se vinculan con algún cuerpo de agua dulce, permanente o temporario, originado por las lluvias y la fluctuación estacional del río Bermejo. Tal como mencionamos, durante la estación lluviosa, los qom recorrían espacios vinculados al monte y los palmares ya que las precipitaciones estacionales garantizaban agua no sólo para ellos sino también para las presas que mariscaban. En cambio, durante la estación seca, la crisis hídrica determinaba que las presas se vincularan a cuerpos de agua permanentes -como el río Bermejo-, razón por la cual los qom circulaban y establecían campamentos en nchaxanaxat. Ya Braunstein (2012: 7) había sugerido la existencia de una polaridad geográfica entre el río y el monte para todos los grupos chaqueños.

Hemos identificado un tercer recorrido llamado Pela'ate' pero en el que no nos detendremos ya que los lugares nombrados que incluye se ubican en zonas muy alejadas de $\mathrm{Km} 503$ a las que no hemos tenido acceso. Este nombre -'zapatos'- remite a que por esta zona se encontraban abandonados calzados hechos con cuero de oso hormiguero (Myrmecophaga tridactyla). Pela 'ate ' parte también de nmiaqa' en dirección al norte y recorre qatec huaỹoxon, peraxanaxa' lamo y elaxarae'la'alqa.

Si bien no será analizado en este trabajo, los tres circuitos mencionados también comprendían sitios ('trojas' o qopi') en los que se depositaban semillas de zapallos y otras hortalizas para ser cosechadas cuando se rehacía el derrotero, así como lugares donde se almacenaban vainas y semillas (principalmente de algarrobo: Prosopis spp.) consumibles, que se aprovechaban cuando se los volvía a visitar en la temporada invernal.

Haremos ahora una breve mención de los caminos que forman parte de los dos primeros circuitos y que son transitados actualmente en los recorridos de marisca.

\section{Caminos (naxasoxonatac)}

Además de estas rutas nacionales, existían caminos considerados equivalentes a "rutas provinciales". Éstos eran más pequeños, más cortos y de menor circulación de gente. Eran y son caminos locales, conocidos y transitados por los qom en sus recorridos de marisca y desconocidos por los habitantes de lugares más distantes. Registramos en Km 503 cuatro caminos: ỹavic, qotaqui, yepachec y nashelte’.

Ỹavic ('quemado') conecta nmiaqa' con el Bermejo, a la altura del topónimo huataxanaq ỹalauat, pasando por la zona actual de vivienda de la comunidad. Su nombre

los que se presentan sequías y algunas heladas (Ginzburg y Adámoli, 2006). 
Tola y Medrano. Circuitos en un espacio nombrado: Toponimia y conocimientos...

remite a que allí se prendió fuego en una ocasión a raíz de la cantidad de hojas y palos secos. Qotaqui ('un tipo de bolsa') es el nombre de otro camino por el que aún transitan y que conecta la punta del madrejón (lalatec, cerca de nmiaqa') con el Bermejo. Su nombre remite a la bolsa que llevan los pescadores al río cuando van de pesca y a que en una ocasión unos pescadores encontraron un qotaqui que había sido olvidado por otros. Yepachec es el nombre de otro camino que va de huaca llegueta al Bermejo. En éste abunda una especie de pasacana o yepat (Harrisia bonplandii); cactus con frutos comestibles. Finalmente, nashelte'va desde nauec late'e hasta el río Bermejo y se lo nombró así porque solía ser recorrido por un anciano que siempre usaba un bastón o nashelte'.

Ahora describiremos algunos de los topónimos que forman parte de los dos primeros circuitos que nos ayudarán a discutir el entramado existente entre los qom y los recursos de subsistencia.

\section{Topónimos de Roquiaxanaxat}

Nmiaqa' era, como mencionamos, el lugar en el que en los meses de fermentación de la algarroba se reunían miembros de las diversas tribus (entre ellas, huaguilot y ỹolo) para celebrar intercambios. En estos encuentros se bailaba el 'baile sapo', una de cuyas finalidades era -como mencionamos- la conformación de parejas y la creación de alianzas. En dichos encuentros participaban algunos pilagás y tobas de otras tribus aliadas. Antes de la década del 80, en nmiaqa' los qom establecían campamento; actualmente es donde se ubica el pueblo criollo que, tras la inundación de 1983, quedó semi-abandonado.

En las inmediaciones existe una laguna en la que relatan que un ser no-humano del agua se llevó a sus profundidades a un niño del grupo de Km 503. Como el abuelo del niño tenía poder para comunicarse con este ser, logró mediar con él y salvarlo. Tal como refieren, en otra oportunidad la dueña de las víboras (araxanaq late'e) impidió que el gobierno realizara un puente en dicha laguna. Hoy en día recurren a ella en busca de agua cuando el madrejón se seca pero sabiendo que allí habitan seres a los que hay que respetar y pedir permiso antes de ingresar.

Huaca llegueta ${ }^{16}$ se ubica sobre el lado norte del Bermejo, en donde éste se hace playo y angosto. Por ahí cruzaban personas y animales de Chaco a Formosa y en esta zona los qom cazaban ñandúes (Rhea americana), nutrias (Myocastor coypus), guazunchos (Mazama spp.), pecaríes (Tayassuidae) y mulitas (Dasypodidae), pescaban, buscaban miel y sacaban curiyú (Boidae), lampalagua (Boidae) e iguana (Tupinambis spp.) para vender su cuero. Según nos relataron, en huaca se juntaban una vez al año con los qom de otras tribus y hacían la fiesta de la peraxanaxa ${ }^{17}$ y bebían aloja (chicha de algarroba). Actualmente es una zona de conflicto con un criollo.

\footnotetext{
${ }^{16}$ Sobre este sitio, ver también un relato registrado por Cristina Messineo (2014).

${ }^{17}$ La peraxanaxa es el palo borracho (Yuchán: Ceiba chodatii) y es el nombre de una fiesta en la que los tobas elaboraban aloja adentro de una batea hecha con el palo borracho y los hombres bebían, bailaban y tocaban música. Estas elaboraciones en grandes bateas se hacían cuando la fiesta preanunciaba gran
} 
Maguisatangui es una laguna en la que pescan con anzuelo y flecha. Abundan las curiyú, los carpinchos (Hydrochaeris hydrochaeris), yacarés (Caiman sp.) y en los montes se encuentra la mi 'ic (ñacanina: Hydrodinastes gigas) de la cual también vendían el cuero. Allí hay pájaros diversos de los cuales los qom extraían los pichones para su cría. También se encontraban árboles especiales para fabricar arcos. De los antiguos qom de Km 503 se sabe que tres ancianos solían hacer campamento en ese lugar. En una oportunidad se les apareció un 'aviaqlec o ser del monte. La permanencia en un sitio de marisca muchas veces dependía del buen trato que los cazadores lograban entablar con los seres no-humanos que allí habitan, dado que no conocer a un humano puede ser motivo de agresiones por parte de los no-humanos y de abandono del lugar por parte de los cazadores.

\section{Lugares nombrados de Nchaxanaxat}

No'onqa coỹo es un campo cercano al Bermejo en el que se encontraba una gran diversidad de pájaros usados como mascotas y con fines medicinales. Desde finales de 1980 es una zona que ocupa un criollo quien, al llegar, cortó todos los árboles de algarrobo. A partir de entonces no hay más pájaros. En este sitio antiguamente los qom hacían campamento.

Nauec copaicalo es un bosque tupido nombrado así porque antiguamente una persona dibujó un gato onza (Leopardus pardalis) sobre la corteza de un árbol (Figura 6).

Figura 6. Nauec copaicalo

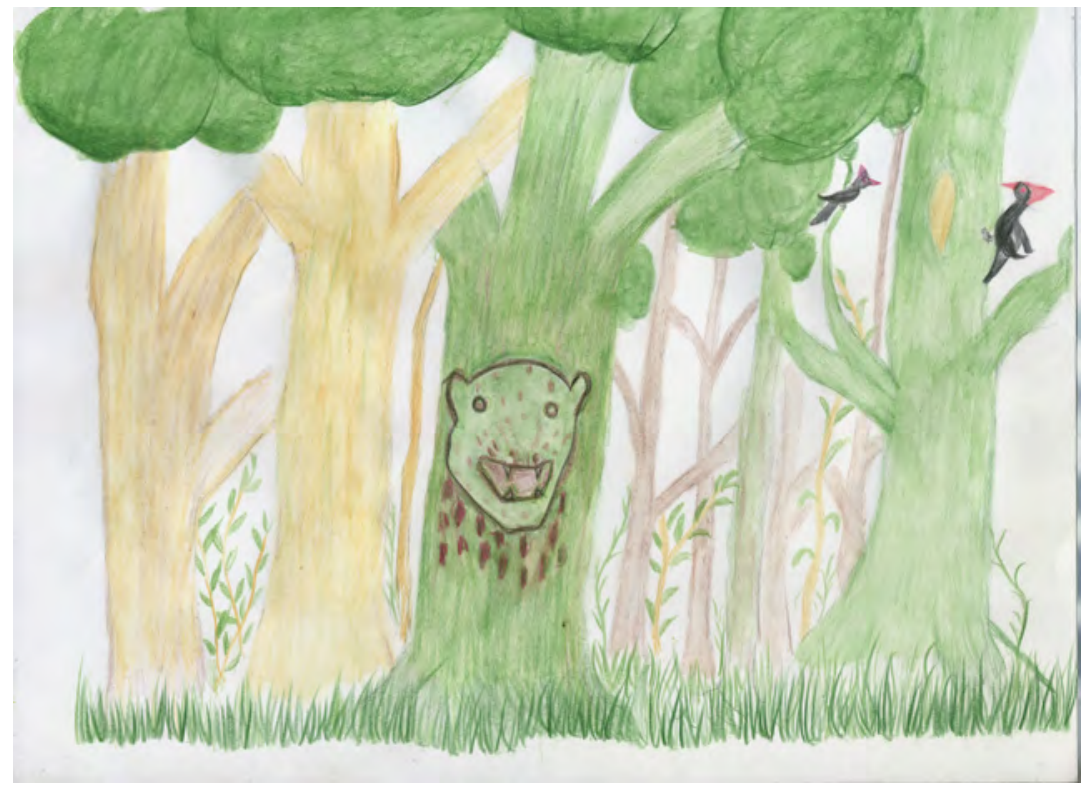

Fuente: Dibujo de Valentín Suárez

concurrencia. 
Tola y Medrano. Circuitos en un espacio nombrado: Toponimia y conocimientos...

Actualmente, este sitio se halla fuera de la tierra de la comunidad, como la mayoría, en una zona ocupada por un criollo. Al lado del monte hay una laguna denominada Lapel saq emapec Loxolqai' que es el nombre en toba de la comunidad y que remite a la existencia de un ser no-humano del agua de cabeza larga que vive allí (Figura 7). Según cuentan los habitantes de Km 503, hacia 1930 existió un poderoso chamán (pi'ioxonaq) llamado Loxolqai' que vivía al borde de la laguna y que podía conversar con el ser de cabeza larga para impedir que se fuera el agua.

Figura 7. Lapel saq emapec Loxolqai

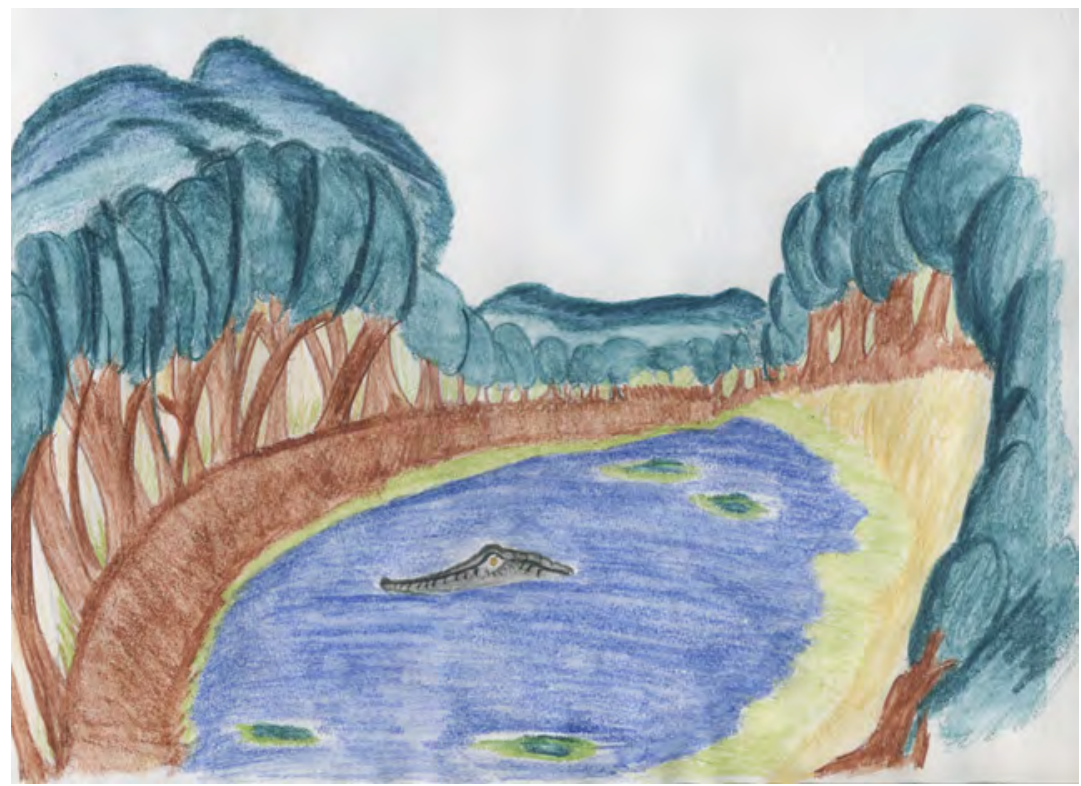

Fuente: Dibujo de Valentín Suárez

Chipiapigui es un gran campo, antiguo lugar de campamento durante los circuitos de marisca. Por las noches, se solía escuchar algo que parecía un relámpago pero que no lo era. Diversos qom de la zona lo conocen por ser allí un lugar de caza de varias tribus. En el centro hay un dellicsat o bosque de palo santo (Bulnesia sarmientoi) pero hasta hace poco era un monte en el que se encontraba una importante diversidad de animales y frutos (amap y pa'atac-algarrobo y algarrobilla: Prosopis spp.-) incluso tigres (Panthera onca). Recientemente, una empresa privada taló el monte y los qom ya no cazan allí.

\section{Mariscando en los circuitos: toponimia y movilidad}

A lo largo de este artículo, a partir de nuestros datos de campo registrados entre los tobas del centro-sur de Formosa, hemos puesto énfasis en dos temáticas principales: por un lado, la articulación entre los circuitos nombrados y la toponimia y, por otro, los 
conocimientos etnoecológicos desplegados en la movilidad propia de dichos circuitos. Los saberes etnoecológicos qom no constituyen un conjunto unificado y homogéneo de conocimientos ambientales de los tobas en su totalidad, sino que son parte del bagaje de conocimientos de grupos de familias localizadas que, a lo largo del tiempo, se vincularon con el entorno, sus animales y sus seres no-humanos. Es por eso que en este artículo hemos tenido en cuenta aspectos de la micro-historia regional ya que la misma es la memoria de los antepasados de los qom con los que hemos trabajado; antepasados que eran parte de unidades sociales localizadas (bandas) que circulaban por la zona central del Chaco argentino aprovechando los recursos que allí había y vinculándose con miembros de unidades sociales mayores (tribus).

La toponimia de las sociedades chaqueñas despertó el interés de numerosos especialistas quienes describieron aspectos diversos de los lugares. Las etnografías realizadas tanto en el Gran Chaco como en otras latitudes mostraron que en sociedades cazadoras-recolectoras el territorio es nombrado en función de las características topográficas y de aquellas que remiten a la presencia de especies animales y vegetales, así como de los acontecimientos que fueron significativos en la memoria de los grupos que crean y transmiten los topónimos. De hecho, los nombres de los lugares y las historias que les están asociadas son transmitidos de generación en generación, estableciéndose así continuidades entre los hechos vividos por los antepasados y la identidad y las prácticas del entorno de los grupos contemporáneos.

Ahora bien, en este texto mostramos que los topónimos del centro-sur de Formosa son parte de circuitos y recorridos más o menos amplios que los tobas transitaban según la época del ciclo anual y en base al conocimiento que tenían del conjunto de las especies animales y vegetales de la región. Ya en la década del 90, Braunstein (1993: 70) mencionó la existencia de caminos que los wichí recorrían y que representaban un óvalo en el que los polos nordeste y suroeste correspondían a las estaciones de invierno y verano, y en los que los campamentos durante el ciclo anual eran reiterados y permanentes. Años después, Wright (2003 a y b) sugirió la existencia de circuitos o rutas tobas, y Mendoza (2003-2004) reconstruyó las movilidades residenciales y logísticas de los tobas del oeste. Braunstein (2012) diferenció también, para los grupos chaqueños en general, entre movilidad a través de circuitos circulares y movilidad a través de circuitos radiales.

En nuestro caso, hemos identificado circuitos circulares nombrados cuya particularidad es que comienzan y terminan en un mismo y único lugar. Estos circuitos, transitados por miembros de diversas tribus, eran recorridos alternadamente según no sólo la abundancia o escasez de recursos de flora y fauna, sino también del agua. Además, los recorridos de marisca actuales y los circuitos completos eran y son explorados de acuerdo a la oposición complementaria monte/agua; dos zonas ambientales transitadas, nombradas y aprovechadas de acuerdo a los conocimientos que los tobas tenían y tienen de la flora, la fauna y del clima. Es por eso que abordamos la movilidad antigua y pasada de los tobas en relación con los diversos conocimientos etnoecológicos que despliegan en su transitar por circuitos y recorridos. Incluso los nombres de los circuitos 
Tola y Medrano. Circuitos en un espacio nombrado: Toponimia y conocimientos...

aluden al conocimiento etnoecológico qom ${ }^{18}$ : ellos son nombrados a partir del nombre del objeto que se usa para la principal actividad de subsistencia realizada en él. Los circuitos hallados son nombrados mediante un proceso metonímico si pensamos que la "metonimia es el proceso mediante el cual se designa una entidad en términos de otra con la que está relacionada por contigüidad (espacial, temporal, causal, instrumental, funcional, etc.)" (Cúneo, 2012: 324, en base a Lakoff y Johnson, 1980: 40). Las denominaciones metonímicas que refieren a los circuitos parecerían responder a un tipo de metonimia por contigüidad en la que el objeto utilizado para la principal actividad realizada en el circuito es usado para nombrar al circuito mismo ${ }^{19}$.

Para cerrar, nuestro análisis de los circuitos nombrados intenta echar luz sobre el modo en que el territorio es recorrido y percibido por miembros de una sociedad de tradición cazadora-recolectora cuya organización social clásica consistía en unidades sociales emparentadas y flexibles. Esto nos remite a lo referido por Luis Cayón para los grupos tukano orientales de Colombia acerca del territorio y la organización social: “... estos pueblos son conscientes del hecho que cada territorio propio se inserta en un contexto mayor, el cual constituye un macro-espacio [...] Muchos lugares del macroespacio pertenecen simultáneamente a varios grupos, señalando los vínculos históricos y culturales de estas sociedades" (2002: 169). Los circuitos de marisca tobas por un amplio territorio nombrado a ambas márgenes del Bermejo dan cuenta de un territorio definido como un macro-espacio que, al ser transitado anualmente por grupos de familias emparentadas que dejaron en él sus huellas y sus memorias generación tras generación, es visualizado menos como un espacio discontinuo o como el simple soporte material en el que se desarrolla la vida, que como una trama multidimensional de recursos y relaciones que, al igual que los circuitos, conecta topónimos, caminos y recorridos, presente y pasado.

Transitando los lugares junto con los qom hemos advertido que la memoria se activa in situ, es decir, que numerosas experiencias de la propia parentela, banda, tribu o grupo de marisca afloran mientras se recorre el territorio y que, en cierto modo, estas historias son leídas en él, constituyendo así la memoria del grupo local. En efecto, aún es posible leer en el territorio la historia de las antiguas tribus y bandas.

\section{Referencias Bibliográficas}

Arenas, P. 2003. Etnografía y alimentación entre los Toba-Nachilamole\#ek y WichiLhuku'tas del Chaco Central. Buenos Aires, edición del autor.

Arenas, P. y Porini, G. 2009. Las aves en la vida de los tobas del oeste de la provincia de Formosa (Argentina). Asunción, Editorial Tiempo de Historia.

Boggiani, G. 1900. Compendio de etnografia Paraguaya Moderna. Asunción.

${ }^{18}$ Los nombres de los caminos, en cambio, remiten a la experiencia de los grupos que recorren dichos caminos, siguiendo la misma lógica de nombramiento de los topónimos.

${ }^{19}$ Los dos nombres de los circuitos (roquiaxanaxat y nchaxanaxat) son derivados instrumentales que, en toba, se construyen mediante el sufijo - axat 'instrumental masculino'. 
Braunstein, J. 1983. "Algunos rasgos de la organización social de los indígenas del Gran Chaco”. En: Trabajos de Etnología, No 2, Universidad de Buenos Aires, pp. 9-102.

1993. "Territorio e historia de los Narradores Matacos". En: Hacia una nueva carta étnica del Gran Chaco, V, Buenos Aires, Las Lomitas, Centro del Hombre Antiguo Chaqueño, pp. 4-74.

2012. "Continuidad histórica de nómades y sedentarios Patrones de asentamiento y procesos de recuperación territorial en el Gran Chaco". En: XXXI Encuentro Nacional de Geohistoria del Nordeste, Universidad Nacional del Nordeste, Resistencia, Argentina.

Braunstein, J. y Miller, E. 1999. "Ethnohistorical Introduction”. En: Elmer Miller (ed) Peoples of the Gran Chaco, Westport, Connecticut, Bergin y Garvey, pp. 1-22.

Buckwalter, A. 2001 [1980]. Vocabulario toba. Formosa/Indiana, Equipo Menonita.

Cayón, L. 2002. En las aguas del Yuruparí: cosmología y chamanismo Makuna. Bogotá, Ediciones Uniandes-Ceso.

Censabella, M. 2009. "Denominaciones etnonímicas y toponímicas tobas: Introducción a la problemática y análisis lingüístico". En: Hacia una nueva carta étnica del gran Chaco, VIII, Buenos Aires, Las Lomitas, Centro del Hombre Antiguo Chaqueño. pp. 213-236.

Cordeu, E. y De los Ríos, M. 1982. "Un enfoque estructural de las variaciones socioculturales de los cazadores-recolectores del gran Chaco". En: Suplemento Antropológico, $\mathrm{N}^{\mathrm{o}} 17$ (1), pp. 131-195.

Cúneo, P. 2012. Clasificación nominal y formación de palabras en toba (familia guaycurú), con especial referencia al léxico etnobiológico. Tesis de doctorado, Buenos Aires, Facultad de Filosofía y Letras, Universidad de Buenos Aires.

Cúneo, P. y Porta, A. 2009. "Vocabulario toba sobre peces y aves". En: Hacia una nueva carta étnica del Gran Chaco, N ${ }^{\circ}$ VIII, Buenos Aires, Las Lomitas, Centro del Hombre Antiguo Chaqueño, pp. 237-252.

De la Cruz, L. M. 1995a. "Thaka honat. Áreas determinadas por los sitios recorridos por las comunidades indígenas del Lote Fiscal 55 de la Provincia de Salta". Hacia una Nueva Carta Étnica del Gran Chaco, No VI, Buenos Aires, Las Lomitas, Centro del Hombre Antiguo Chaqueño, pp. 65-68.

1995b. "Qomlajépi naleua, nuestra tierra. Los sitios que contienen la tierra que da vida a los tobas de Sombrero Negro de la provincia de Formosa". Hacia una Nueva Carta Étnica del Gran Chaco, N ${ }^{\circ}$ VI, Buenos Aires, Las Lomitas, Centro del Hombre Antiguo Chaqueño. pp. 69-114.

Dell'Arciprete, A. 1991. "Lugares de los Pilagá". Hacia Una Nueva Carta Etnica del Gran Chaco, $\mathrm{N}^{\circ}$ II, Buenos Aires, Las Lomitas, Centro del Hombre Antiguo Chaqueño, pp. 58-84.

Dickens, G. 1938. "Fishing on the Pilcomayo". En: The Magazine of the South American Missionary Society, № LXXII (813), pp. 55-56. 
Fernández, A. y Braunstein, J. 2001. "Historias de Pampa del Indio”. En: IV Congreso Argentino de Americanistas, Buenos Aires, Sociedad Argentina de Americanistas, ed. Dunken, Tomo 2, pp. 161-193.

Ginzburg, R. y Adámoli, J. 2006. "Situación ambiental en el Chaco Húmedo". En: Brown, A., U. Martinez Ortiz, M. Acerbi y J. Corcuera (eds) La Situación Ambiental Argentina 2005, Buenos Aires, Fundación Vida Silvestre Argentina, pp. 103-113.

Iriondo, M. 2006. "Cambios ambientales en el Chaco argentino y boliviano en los últimos miles de años". En: Folia Histórica del Nordeste, Resistencia, No 16, Resistencia, Instituto de Investigaciones Geohistóricas-CONICET, Instituto de Historia-UNNE, pp. 39-50.

Karsten, R. 1932. Indian tribes of the Argentine and Bolivian Chaco. Helsingfors, Societas Scientiarum Fennica.

Kersten, L. 1968 [1905]. Las tribus indígenas del Gran Chaco hasta fines del siglo XVIII. Resistencia, Universidad Nacional del Nordeste.

Lamenza, G. 2013. El hombre y el ambiente en el Holoceno Tardio del Chaco Meridional. Catamarca, Universidad Nacional de Catamarca.

Martínez, G. 2007. "La farmacopea natural en la salud materno-infantil de los Tobas del Río Bermejito". En: Kurtziana, N 33, pp. 39-63.

2008. La farmacopea natural en la etnomedicina de los Tobas del Río Bermejito (Chaco, Argentina). Tesis Doctoral, Córdoba, Facultad de Ciencias Agropecuarias, Universidad Nacional de Córdoba.

2009a. "Las denominaciones vernáculas y el conocimiento toba del entorno vegetal”. En: Revista de Dialectología y Tradiciones Populares, N ${ }^{\circ}$ LXIV (2), pp. 149-168.

2009b. "Fitonimia de los Tobas Bermejeños (Chaco Central, Argentina)". En: Hacia una nueva carta étnica del gran Chaco, N ${ }^{\circ}$ VIII, Buenos Aires, Las Lomitas, Centro del Hombre Antiguo Chaqueño, pp. 194-212.

2010. "Enfermedad y entidades anímicas del entorno natural. Etiologías religioso-rituales y espacios ambientales entre los tobas del Chaco Central, Argentina”. En: Revista de Antropología Iberoamericana, № 5 (2), pp. 189-221.

2011. "Uso de plantas medicinales en el tratamiento de afecciones transmitidas por el agua en una comunidad toba (qom) del Impenetrable (Chaco, Argentina): Una perspectiva etnoecológica y sanitaria". En: Bonplandia, $\mathrm{N}^{\mathrm{o}} 20$, pp. 329-352.

2013. "Saberes locales y promoción de la salud ambiental entre los tobas (qom) del Chaco Central (Argentina). Trayectos de una investigación etnoecológica en contextos de interculturalidad". En: X Reunión de Antropología del Mercosur, Universidad Nacional de Córdoba, Julio 10-13, 2013; Córdoba, Argentina.

Martínez Crovetto, R. 1964. "Estudios etnobotánicos I. Nombres de plantas y su utilidad, según los indios tobas del este del Chaco". En: Bonplandia, Nº 1, pp. 279-333. 
1965. "Estudios etnobotánicos. II. Nombres de plantas y su utilidad, según los indios Vilelas del Chaco”. En: Bonplandia, N² 2, pp. 1-23. 1995. Zoonimia y etnozoología de los pilagá, toba, mocoví, mataco y vilela. Buenos Aires, Universidad de Buenos Aires, Facultad de Filosofía y Letras, Archivo de Lenguas Indoamericanas, Colección Nuestra América, Instituto de Lingüística.

Medrano, C. 2012. Zoo-sociología qom: de cómo los hombres y los animales trazan sus relaciones en el Gran Chaco. Tesis de Doctorado, Buenos Aires, Facultad de Filosofía y Letras, Universidad de Buenos Aires.

2013. "Devenir-en-transformación: debates etnozoológicos en torno a la metamorfosis animal entre los qom". En: Tola, F., C. Medrano y L. Cardín (eds) Gran Chaco. Ontología, poder, afectividad, Buenos Aires, Rumbo Sur, pp. 77-101.

2014. “Zoo-sociocosmología qom: seres humanos, animales y sus relaciones en el Gran Chaco". En: Journal de la Société des Américanistes, No 100 (1), pp. 225-257.

Medrano, C.; Maidana, M. y Gómez, C. 2011. Zoología Qom. Conocimientos tobas sobre el mundo animal. Santa Fe, Ediciones Biológica, Serie Naturaleza, Conservación y Sociedad.

Mendoza, M. 2002. Band Mobility and Leadership Among the Western Toba HunterGatherers of the Gran Chaco in Argentina. New York, Edwin Mellin Press.

2003-2004. "Range area and seasonal campsites of Toba bands in western Chaco, Argentina”. En: Before farming, N4 (2), pp. 1-12.

Messineo, C. 2014. Arte verbal qom. Consejos, rogativas y relatos. Buenos Aires, Rumbo Sur/Ethnographica.

Métraux, A.1963 [1944]. "Ethnography of the Chaco". En: J. Steward (ed.) Handbook of South American Indians, № 1 (2), New York, Cooper Square Publishers Inc., pp. 197-380.

1996 [1946]. Etnografía del Chaco. Paraguay, El Lector.

Miller, E. 1979. Los tobas argentinos. Armonía y disonancia en una sociedad. México, Siglo XXI.

Morello, J. H.; Rodriguez, A. F. y Silva, M. 2009. "Clasificación de ambientes en áreas protegidas de las ecorregiones Del Chaco húmedo y seco”. En: Morello, J. H. y Rodríguez, A. F. (eds.) El Chaco sin bosques: la Pampa o el desierto del futuro, Buenos Aires, Orientación Gráfica, pp. 53-91.

Palavecino, E. 1936. "Las culturas aborígenes del Chaco". En: De la Historia de la Nación Argentina, vol. I, Buenos Aires, Junta de Historia y Numismática Americana, Imprenta de la Universidad, pp. 429-472.

Palmer, J. 1995. "Wichi toponymy”. Hacia una Nueva Carta Étnica del Gran Chaco, No VI, Buenos Aires, Las Lomitas, Centro del Hombre Antiguo Chaqueño, pp 3-64.

Salamanca, C. 2011. Movilizaciones indigenas, mapas e historias por la propiedad de la tierra en el Chaco Argentino. La lucha de las familias tobas por Poxoyaxaic alhua. Buenos Aires, FLACSO, IWGIA, CONICET. 
Tola y Medrano. Circuitos en un espacio nombrado: Toponimia y conocimientos...

Scarpa, G. y Pastor, A. 2004. "Vegetation units of the Argentine Semiarid Chaco: The Toba-Pilagá perception". En: Phytocoenologia, No34 (1), pp. 133-161.

Tola, F. 2009. Conceptions du corps et de la personne dans un contexte amerindien: les toba du Gran Chaco. París, L'Harmattan.

2010. "Maîtres, chamanes et amants. Quelques réflexions sur la conception toba de l'agentivité". En: Ateliers du LESC, 34: http://ateliers.revues.org/8538 [capturado: 27/9/10].

2012. Yo no estoy solo en mi cuerpo. Buenos Aires, Biblos.

Tola, F.; Medrano, C. y Cardin, L. 2011. Territorio, memoria y lengua entre los qom del centro-este de Formosa. Buenos Aires, Edición de las autoras.

Toledo, V. 1992. "What is Ethnoecology? Origins, scope and implications of a rising discipline”. En: Etnoecológica, No 1 (1), pp. 5-21.

Toledo, V. M.; Alarcón-Chaires, P.; Moguel, P.; Olivo, M.; Cabrera, A.; Leyequien, E. y Rodríguez-Aldabe, A. 2001. "El Atlas Etnoecológico de México y Centroamérica: Fundamentos, Métodos y Resultados”. En: Etnoecológica, Vol. 6, № 8, pp. 7-41. Vuoto, D. L. 1981a. Aspectos de la interrelación entre la fauna y la cultura Toba-Taksek. Tesis de Licenciatura, Buenos Aires, Facultad de Filosofía y Letras, Universidad de Buenos Aires.

1981b. "La fauna de los Toba-Taksek". En: Entregas del Instituto de Tilcara, $\mathrm{N}^{\mathrm{0}}$ 10, Tilcara, pp. 77-138.

Vuoto, P. 1981. "Plantas útiles entre los toba-taksek". En: Entregas del Instituto de Tilcara, $\mathrm{N}^{\mathrm{o}} 10$, Tilcara, pp. 12-76.

1999. "Recolección y poder. La vegetación entre los toba del este de Formosa". En: Aschero Carlos, Vuoto Patricia y Korstanje Alejandra (eds) En los tres reinos: prácticas de recolección en el Cono Sur de América, Tucumán: Instituto de Arqueología y Museo, Facultad de Ciencias Naturales e Instituto Miguel Lillo, Universidad Nacional de Tucumán, pp. 239-250.

Wright, P. 1991. "Topónimos de la zona de Misión Tacaaglé (Formosa)”. En: Hacia una Nueva Carta Étnica del Gran Chaco, No II, Buenos Aires, Las Lomitas, Centro del Hombre Antiguo Chaqueño, pp. 41-57.

2003a. "Entre La marisca y el trabajo. Modos indígenas de apropiación del medio ambiente". En: Cordeu Edgardo, Fernández Analía; Messineo Cristina; Ruiz Moras Ezequiel y Wright Pablo (eds) Memorias Etnohistóricas del Gran Chaco. Etnias toba (qom) y chamacoco (ishir), Buenos Aires, Taller 4 Ediciones, pp. 99-119.

2003b. "Textos orales referentes a los circuitos, lugares y prácticas de marisca de los toba”. En: Cordeu Edgardo, Fernández Analía; Messineo Cristina; Ruiz Moras Ezequiel y Wright Pablo (eds) Memorias Etnohistóricas del Gran Chaco. Etnias toba (qom) y chamacoco (ishir), Buenos Aires, Taller 4 Ediciones, pp. 551-554.

2008. Ser-en-el-sueño. Crónicas de historia y vida toba. Buenos Aires, Editorial Biblos/Culturalia. 